\title{
¿Por qué tenemos que reevaluar el consentimiento en el contexto de la violencia sexual?
}

\author{
Why we need to review consent in the context of sexual \\ violence?
}

\author{
IVÁN WiLLIAMS JiMÉNEZ \\ Institution of Occupational Safety and Health, \\ Leicester, United Kingdom \\ Investigador en Universidad Carlos III de Madrid \\ ORCID: https://orcid.org/0000-0003-0274-6079
}

Recibido: $12 / 6 / 2019$

Aceptado: 22/11/2019

doi: https://doi.org/10.20318/femeris.2020.5161

Resumen. La violencia sexual está ampliamente arraigada y es un problema sistémico a nivel mundial. En su tratamiento jurídico esta problemática todavía es trivializada y carece de la debida relevancia en la agenda normativa. El incremento de sucesos mediáticos y una mayor visibilizacion de debates públicos en relación con el consentimiento en el contexto de la violencia sexual demuestran que existe una imperiosa necesidad de redefinir que constituye el consentimiento en el marco jurídico. Este término con un marcado enfoque de género todavía se enmarca en un espacio relacionado con la violencia sexual que no ha sido deliberadamente analizado por parte del legislador ni acompasado con los actuales tiempos.

En este ámbito los legisladores han estado históricamente predispuestos por estereotipos culturales y normas sociales impuestas que han limitado un progreso natural en cómo se entiende e interpreta el consenso. La necesidad de abordar este debate a nivel nacional viene determinada por una mayor casuística existente en otras jurisdicciones (en su gran mayoría a nivel europeo) en la definición por parte de la ley sobre que es el consentimiento sexual. Ante esta situación cabe cuestionarse, ¿está el marco jurídico preparado para abordar esta problemática?

Palabras clave: consentimiento, violencia sexual, abuso sexual, violencia de género.

Abstract. Sexual violence is widespread and systemic worldwide ${ }^{* *}$. At a legal level this issue still remains trivialised and largely neglected from the regulatory agenda. The increasing amount of protests and public debates following particular cases on issues related to consent in the context of sexual violence demonstrate the urgent need to redefine what constitutes sexual consent in the legal context. This term that entails a gender-based sexual violence connotations still lacks from clarity from the perspective of a collective understanding. As a pervasive issue it still requires fit for purpose regulations and to keep pace with times.

\footnotetext{
*ivan.williams78@gmail.com

${ }^{* *}$ Amnesty International. Right to be free from rape overview of legislation and state of play in Europe and international human rights standards, 2018
} 
In the legal scene legislators have been historically biased by cultural imposed stereotypes and social norms that have limited a natural progress in the way consent is understood and interpreted. The need to address this legal debate at a national is justified by an increasing progress made in other jurisdictions (significant progress in European jurisdictions) in the definition of sexual consent in law. In order to address the issue the question that's worth formulating is if the current regulatory framework for sexual consent law is appropriate from a legal standpoint.

Keywords: consent, sexual violence, gender-based violence.

Este artículo pretende poner de relieve donde se encuentran las principales discrepancias en el tratamiento jurídico del consentimiento sexual y sus implicaciones a nivel legal.

Las cuestiones legales acerca del consentimiento sexual están adquiriendo una mayor relevancia en el plano jurídico. En este sentido, la problemática a nivel internacional se ha caracterizado por legislación inadecuada carente de efectividad y por una falta de estandarización en la definición de consentimiento. Estas cuestiones están causando inconsistencias en el tratamiento y abordaje de la materia y dificultad en la forma en la que se dirime esta problemática.

Por un lado, las citadas carencias en el plano jurídico justifican la necesidad de cambios en la legislación como mecanismo para transformar actitudes que normalmente se han visto influenciados por estereotipos sexistas y malinterpretaciones sobre la violencia sexual. Estos prejuicios han estado presentes en las diferentes figuras jurídicas relacionadas con esta materia. Esto, sin duda, ha ayudado a perpetuar un denotado vacío legal en las prácticas habituales de litigación, regulación y judicialización que aborda la problemática del consentimiento.

A este respecto, cabe resaltar que las leyes y estándares internacionales obligan a los diferentes estados a promocionar cambios en los patrones de comportamiento sociales y culturales de los hombres y las mujeres con miras a la erradicación de los prejuicios, las costumbres, las tradiciones y cualquier otra práctica que estén basadas en la idea de la inferioridad de la mujer o de roles de estereotipos ${ }^{1}$. Esta obligación ha sido articulada a nivel europeo, donde se ha registrado un aumento del número de países con definiciones basadas en la figura del consenso, o donde el consenso está de alguna forma presente en las leyes y sanciones penales².

No obstante, todavía no parece claro y se antoja complicado de entender que es lo que la ley considera ilegal cuando se trata de analizar si la participación y el consentimiento es voluntario o no, o si existe un consentimiento expreso a través de palabras, actos, o de alguna otra manera. Esta área gris tiene un impacto considerado en el tratamiento jurídico y en la protección de las víctimas. Supone sin duda una barrera más, cuando se trata de restringir la violencia masculina en contra de la mujer. Aquí la ley de cierta manera consiente esta situación, a través de una ausencia de prohibición legal expresa de las diferentes formas de violencia sexual ${ }^{3}$.

\footnotetext{
${ }^{1}$ Convención de Estambul. Artículo 12 (1).

${ }^{2}$ Ivan Williams. Equality Now. The World's Shame. The Global Rape Epidemic. How Laws around the World are failing to protect Women and Girls from Sexual Violence, 2017, p 13.

${ }^{3}$ Franks, Mary Anne. Men, women, and optimal violence. University of Illinois Law review. 2016
} 
A nivel jurídico en ocasiones parece que existe un interés deliberado en que esta falta de claridad continúe. Los límites relacionados con la actividad sexual deben ser reconsiderados de manera periódica, y características como la pasividad o la ausencia de violencia expresa no deben ser consideradas como un signo de participación voluntaria. A este respecto, no debería existir una presunción en la ley que dictamine que la víctima da su consentimiento porque no ha mostrado una resistencia física a una determinada conducta sexual no deseada, sin importar si el autor amenazó con hacer uso o no de violencia física. ${ }^{4}$ Por tanto, no debería existir asunción alguna en el derecho o en la práctica que dictamine que una víctima da su consentimiento porque no se resistió física o activamente contra la conducta sexual no deseada, y sin importar o no si el responsable amenazó con hacer uso de violencia física.

El otro aspecto clave a destacar es la cuestión relacionada con el significado de la terminología sobre la figura del consentimiento sexual. De acuerdo con las regulaciones de derechos humanos internacionales, la violación, junto con otras formas de violencia sexual deben ser definidas como conductas sexuales sin el consentimiento de la víctima. Esto incluye el uso de la coerción, de medios violentos o no como es el uso de la fuerza, la mera amenaza de fuerza, temor a la violencia, detención, opresión psicológica o abuso de poder. También incluye los supuestos en los que en los que la voluntariedad de participar en actos sexuales no fuera dada de manera libre y verdadera; o cuando la víctima no sea capaz de dar un consentimiento genuino porque estuviera drogada, inconsciente o no tuviera la capacidad mental para consentir ${ }^{5}$.

Las definiciones por parte del legislador pueden estar abiertas a diferentes interpretaciones. Además, aspectos como el incremento del uso de la tecnología en la violencia sexual no hacen sino agravar esta ambigüedad 6 . Si se pretende hacer del consenso algo crucial en las conversaciones sexuales y que la legislación se mantenga al día de los tiempos actuales, prácticas emergentes como es la publicación de imágenes íntimas sin consentimiento también requerirán de una mayor atención desde una perspectiva legal.

Las inconsistencias en la definición de consentimiento sexual son sin duda un aspecto clave, ya que pueden determinar que comportamientos constituyen o no agresión sexual y violación. Esta falta de consistencia ha sido destacada por numerosos autores ${ }^{7}$, centrando la problemática en dos aspectos clave: la falta de acuerdo sobre si el consentimiento debe ser mostrado en condiciones donde se ejerza la coerción y si este debe ser verbal o no ${ }^{8}$. Este aspecto es de nuevo complejo en su tratamiento legal, ya que muchos modelos se basan en la existencia del consenso por defecto en aquellas situaciones en las que no exista una negativa expresa a participar en un acto sexual en lugar de interpretar el consenso como una participación y como un gesto afirmativo ${ }^{9}$.

\footnotetext{
${ }^{4}$ Iván Williams. International Criminal Court. Artículo 7(1)-(g)1(1)

${ }^{5}$ Amnesty International. Right to be free from rape overview of legislation and state of play in Europe and international human rights standards, 2018.

${ }^{6}$ Kyana D. Dixie . Defining Consent as a Factor in Sexual Assault Prevention, 2017.

${ }^{7}$ Beres, M. A. Spontaneous sexual consent: An analysis of sexual consent literature. Feminism \& Psychology, 17 (1), 93-108.

${ }^{8}$ Eleanor M. McGrath. Let's Talk About (Consensual) Sex!. 2016.

${ }^{9}$ Amnesty International. Right to be free from rape overview of legislation and state of play in Europe and international human rights standards, 2018.
} 


\section{Trabajos citados}

IVÁN WILLIAMS. Right to be free from rape overview of legislation and state of play in Europe and international human rights standards, 2018

CONVENCIÓN DE ESTAMBUL. Artículo 12 (1)

EQUALITY NOW. The World's Shame. The Global Rape Epidemic. How Laws around the World are failing to protect Women and Girls from Sexual Violence, 2017, p 13.

FRANKS, MARY ANNE. Men, women, and optimal violence. University of Illinois Law review. 2016

INTERNATIONAL CRIMINAL COURT. Artículo 7(1)-(g)1(1)

AMNESTY INTERNATIONAL. Right to be free from rape overview of legislation and state of play in Europe and international human rights standards, 2018.

KYANA D. DIXIE . Defining Consent as a Factor in Sexual Assault Prevention, 2017.

M. A. Spontaneous sexual consent: An analysis of sexual consent literature. Feminism \& Psychology, 17 (1), 93-108.

ELEANOR M. MCGRATH. Let's Talk About (Consensual) Sex!. 2016.

AMNESTY INTERNATIONAL. Right to be free from rape overview of legislation and state of play in Europe and international human rights standards, 2018. 